\section{OCCUPATIONAL EXPOSURE TO CHLORINATED SOLVENTS AND LUNG CANCER: RESULTS FROM THE ICARE STUDY}

${ }^{1}$ Francesca Mattei, ${ }^{1}$ Florence Guida, ${ }^{1}$ Marie Sanchez, ${ }^{1}$ Sylvie Cénée, ${ }^{2}$ Joëlle Févotte, ${ }^{3}$ Daniele Luce, 'Isabelle Stücker. 'Inserm, CESP Centre for Research in Epidemiology and Population Health, U1018, Environmental Epidemiology of Cancer Team, Villejuif, France; ${ }^{2}$ Inserm, CESP Centre for Research in Epidemiology and Population Health, U1018, Epidemiology of Occupational and Social Determinants of Health Team, Villejuif, France; ${ }^{3}$ UMRESTTE (Unité Mixte de Recherche Épidémiologique Et de Surveillance en Transport, Travail Et Environnement), University Claude Bernard, Lyon, France

\subsection{6/oemed-2014-102362.52}

Objectives We aimed to investigate the role of occupational exposure to chlorinated solvents in the aetiology of lung cancer. Method ICARE is a multicenter population-based case-control study conducted in France between 2001 and 2006. Information on subjects lifelong work history was collected by face to face interviews using standardised questionnaires. Occupational exposures were assessed using job-exposure matrices (JEM) relative to five chlorinated solvents including trichloroethylene (TCE), methylene chloride, perchloroethylene (PER), chloroform and carbon tetrachloride. Solvents were studied separately and since overlapping among exposures analyses for combined solvents exposure were performed. In the questionnaire, subjects also had to report if they were exposed to TCE or other substances (PER was among them). Odds ratios (ORs) were computed using unconditional logistic regression models adjusted for classical risk factors. Results A total of 2926 cases (2276 men and 650 women) and 3555 controls ( 2780 men and 775 women) were included. A statistically significant positive association for lung cancer risk was observed in both men (OR 1.47, 95\% CI: 1.00-2.17) and in women (OR 3.86, 95\% CI: 1.36 -11.01) exposed to PER combined with TCE and/or methylene chloride. In contrast, no statistically significant associations were found for TCE or other solvent combinations. Finally for subjects, who reported the exposure to PER, the ORs were 3.25 (95\% CI: 1.23, 8.59) and 3.12 (95\% CI: $0.50,19.28)$ among men and women respectively.

Conclusions The results of this study suggest that PER alone or in combination with TCE and/or methylene chloride may increase the risk of lung cancer.

\section{RISK OF TOTAL HIP REPLACEMENT IN RELATIONSHIP TO CUMULATIVE EXPOSURES IN THE WORK ENVIRONMENT}

${ }^{1,2}$ Tine Steen Rubak, ${ }^{3}$ Susanne Wulff Svendsen, ${ }^{2}$ Poul Frost. 'Department of Occupational Medicine, Slagelse Hospital, Slagelse, Denmark; ${ }^{2}$ Danish Ramazzini Centre, University Department of Occupational Medicine, Herning Regional Hospital, Herning, Denmark; ${ }^{3}$ Danish Ramazzini Centre, Department of Occupational Medicine, Aarhus University Hospital, Aarhus, Denmark

\subsection{6/oemed-2014-102362.53}

Objectives To investigate the risk of total hip replacement (THR) due to primary osteoarthritis (OA) in relation to cumulative occupational mechanical exposures and lifestyle factors.

Method In a population of Danish employees, we identified first-time THR cases in the National Patient Register. For each case, two age- and sex-matched controls were drawn. In total 2500 randomly selected case-control sets were mailed a questionnaire entailing job history, weight (present and at age 25), height, smoking and sports activities at age 25. We used a job exposure matrix to calculate cumulative exposures in the work environment. Exposure estimates were expressed according to the pack-year concept of smoking (cumulative lifting $=$ tonyears). We used conditional logistic regression.

Results $71 \%$ of case-control sets contributed to the study. The adjusted odds ratio for exposure to at least 20 ton-years was 1.35 (95\% CI 1.05-1.74) for men. When analysing frequent lifting as risk factor, the adjusted OR reached 1.26 (95\% CI 0.99 1.61). There were no such increases for women. We controlled for body mass index, sports participation at age 25 and previous trauma towards the knees.

Conclusions When using a job exposure matrix we found a modest increased risk of THR in relation to cumulative lifting for men. For women no increased risk was seen.

\section{MEDIATING EFFECTS OF HEALTH ON THE ASSOCIATION BETWEEN NEGATIVE LIFE EVENTS IN CHILDHOOD ON FUTURE LABOUR MARKET PARTICIPATION. A 7-YEAR FOLLOW-UP STUDY}

1,2 Merete Labriola, ${ }^{2} J o h a n$ Hviid Andersen, 1,3Thomas Lund. 'MarselisborgCentret, Research and Development Public Health and Quality Improvement Central Denmark Region, Aarhus, Denmark; 'Danish Ramazzini Centre, Department of Occupational Medicine, Regional Hospital Herning, Herning, Denmark; ${ }^{3}$ National Centre for Occupational Rehabilitation, Rauland, Norway

\subsection{6/oemed-2014-102362.54}

Objectives The aim of this study was to investigate if effects of multiple negative life events in childhood on future labour market participation were mediated through 3 measures of mental, general, and psychosomatic health.

Method Of a cohort of 3681 born in 1989 in the county of Ringkjoebing, Denmark, 3058 (83\%) completed a questionnaire in 2004. They were followed in a register on social benefits for 12 months in 2010-2011. Logistic regression analyses were used to investigate associations between negative life events in early childhood and future labour market participation at age 21-22. Sobel-Goodman test for mediating effects was used to determine, if part of the effects were mediated through mental, general and psychosomatic health at age 14/15.

Results Labour market participation decreased with increase in negative life events, especially for females. However, for females, only a small proportion of this effect was mediated through health: $1 \%$ through psychosomatic symptoms, $4 \%$ through general self-rated health, and $4 \%$ through mental health. For males, a larger proportion of the effects were mediated through health: $1 \%$ through psychosomatic symptoms, $8 \%$ through general selfrated health, and 14\% through mental health.

Conclusions Information on childhood conditions may increase the understanding of determinants of labour market participation for young adults. Only a small proportion of the negative effects of childhood adversities were mediated through health among females. For males, the results suggest that childhood adversities affect especially mental health to a degree where it threatens future labour market status.

\section{MENTAL HEALTH IN CHILDHOOD AS RISK INDICATOR OF LABOUR MARKET PARTICIPATION IN YOUNG ADULTHOOD. A PROSPECTIVE BIRTH COHORT STUDY}

1,2Thomas Lund, 'Johan Hviid Andersen, 1,3Merete Labriola. 'Danish Ramazzini Centre, Department of Occupational Medicine, Regional Hospital Herning, Herning, Denmark; ${ }^{2}$ National Centre for Occupational Rehabilitation, Rauland, Norway; ${ }^{3}$ MarselisborgCentret, Research and Development Public Health and Quality Improvement Central Denmark Region, Aarhus, Denmark

10.1136/oemed-2014-102362.55 